\title{
Analysis of Medical Interventions due to Toxic Effects of Contacts with Animals, Including Envenoming (X20-X27 and T63 ICD-10) in Lower Silesia Region of Poland in Years 2006-2012
}

\author{
Jaroslaw Drobnik ${ }^{1}$, Piotr Nowicki ${ }^{2}$, Robert Suslo ${ }^{1}$ \\ ${ }^{1}$ Public Health Department, Clinical University Hospital, Wroclaw, Poland \\ ${ }^{2}$ Management, Autonomous Clinical Hospital, Wroclaw, Poland \\ robertsuslo@gmail.com
}

\begin{abstract}
Despite of ongoing civilization progresses there are numerous cases of human intoxications resulting from interactions with animals. Data on causes of hospital admissions secondary to contacts with venomous animals and subsequent toxic effects (X20-X27 and T63 according to ICD-10) in the years 2006-2012, made available due to courtesy of the public healthcare insurance provider Narodowy Fundusz Zdrowia in Lower Silesia region of Poland (NFZ), were analyzed. The aim of the retrospective study was to reveal current incidence and trends in the most common and important contacts with venomous animals in the analyzed time period as knowing it can facilitate the process of diagnostics for both clinical and forensic medicine purposes. The study revealed that the most common treated in hospital health problems resulting from contacts with animals were associated not with vertebrate but with arthropods - especially hornets, wasps, bees and arthropods other than scorpions and spiders; there was a significant rising trend in such contacts occurring at humans' homes. The rising trend in hospital admissions due to toxic effects of snake venom shall be attributed in the first place to the growing popularity of tourism practiced on foot in areas inhabited by snakes. The rapid increase in hospitalizations number due to toxic effects of contact with scorpion and venomous fishes results most probably from keeping them as pets at private houses as they are absent among natural Polish fauna. It is very difficult to detect envenoming or toxic effect of contact with an unknown exotic animal - so it is crucial forforensic medicine practitioners to gain information from clinical history, witnesses' reports and scene evaluation. At the national level there is the need for creation of an animal toxic substances reference standards bank - serving for the purposes of both clinical and forensic toxicological analyses.
\end{abstract}

Keywords: Forensic Medicine; Forensic Toxicology; Envenoming; Toxic Animal

Abbreviations: NFZ - Narodowy Fundusz Zdrowia in Lower Silesia region of Poland, District Division in Wroclaw

\section{INTRODUCTION}

In order to treat cases of toxic effects resulting from venomous animals bites properly, human medicine had to adapt knowledge accumulated by veterinary sciences, especially recent progresses made by veterinary pathology [1]. The majority of venomous organisms, snakes included, reside in the southern parts of Europe [2]. In Poland there are not many domestic venomous organisms among snakes there is only one such species: the venomous adder (Vipera berus) [3]. It inhabits practically whole territory of Poland, living most often on the borders of woods [4]. However, it must be taken into account that more and more often the wild animals re-colonize humans' nearest environment, including centers of cities and even the most modern buildings [5]. Cases of Vipera berus bites are most commonly reported in the southwestern regions of Poland. Snake bite can trigger a wide spectrum of symptoms: they may be limited to mild local edema that lasts for several weeks but can also encompass severe systemic disturbances, including: shock, prolonged hypotension, bronchospasm and laryngeal edema, diarrhea, cardiac arrhythmias, neutrophilic hyperleukocytosis and thrombocytopenia. Specific anti-venom is available but it is not needed to be administered in all cases of Vipera berus bites [4]. However, a single dose of the specific anti-venom administered within the first 10 hours after the bite incident significantly reduces incidence of hematomas in the wound region, as well as the discomfort intensity and the length of hospital stay [6]. The specific treatment 
hast to be started as soon as possible to be efficient so the pattern of lesions typical for snake bite needs to be properly and rapidly recognized by all medical professionals [7]. The vast majority of Vipera berus bite cases in Poland are recorded in June, July and August - most often they are located on the hand or foot; deaths are rare [8]. However, as Vipera berus venom demonstrates proteolytic, fibrynolytic, anticoagulant and phospholipase activity, all patients bitten by it should be admitted to hospital for observation [9]. Contacts with animals cause serious envenoming comparatively rarely; far more common are less drastic but typically still bothersome syndromes - the common example is contact with hornets, wasps or bees. Among the most serious complications are local allergic reactions to insects' venoms - they occur even in nearly $20 \%$ of the general population and systemic oversensitivity reactions are recorded in nearly 5\% of the victims [10]. In Poland wild bees are quite rare and - luckily - the problem of "killer bees" is nonexistent - while it occurs in many other countries of the world [11]. It becomes more and more popular these days to keep exotic animals as pets, among others: snakes, spiders, scorpions, fishes or even marine invertebrates - that quite often turn out being dangerous to owners, their families and neighbors [12]. Envenoming from untypical species can be live threatening even in cases when specific anti-venom serum exists - as only on a rare basis it is readily available at the desired place and in the needed amount. As the commercial sources are inadequate regional state-driven anti-venom serum banks may be the rights solution [13]. Screening for unknown compounds - including untypical animal toxic substances - is difficult and sometimes identifying them without some preexisting clues turns out to be impossible at all, especially under tight time constraints [14]. Snake bites are in the most part accidental, suicides by snake bite are very rare, and only historical sources provide information on capital punishment executions performed by throwing the victim into a hole full of snakes. Recently cases of homicides with the use of snakes were reported that were carried out by intentionally exposing the victim to venomous snakes bite $[15,16]$. Such a theoretical possibility was pointed at in medico-legal scientific papers already several years ago [17].

\section{MATERIALS AND MethodS}

Data on causes of hospital admissions caused by contacts with venomous animals and subsequent toxic effects (X20-X27 and T63 according to the International Classification of Diseases revision 10 (ICD-10), respectively) in the years 2006-2012, made available due to courtesy of the public healthcare insurance provider Narodowy Fundusz Zdrowia in Lower Silesia region of Poland (NFZ), were analyzed. The data included all admissions to hospital regardless of the subsequent hospital stay duration length. The aim of the study was to reveal the current incidence and trends in the most common and important contacts with venomous animals in the analyzed time period - in order to facilitate the diagnostics process for both clinical and forensic medicine purposes.

\section{RESULTS AND DISCUSSION}

During the years 2006-2012 the number or age structure of the Lower Silesia region of Poland population did not undergo significant changes; however some effects of population ageing were observed [18]. In the year 2014 in the Lower Silesia there were living 2,9 million people, $52 \%$ of them females [19].

In the years 2006-2012 there were total 29527 cases of toxic effects of contacts with various animals (X20-X27 and T63-T63.9 according to ICD-10) resulting in hospital admissions in the Lower Silesia region of Poland - which translates into around 4200 of such medical interventions yearly on average.

The study revealed that in the years 2006-2012 there were total 1860 admissions to hospitals of the Lower Silesia region of Poland due to not otherwise specified toxic effects of contact with venomous animals (T63 according to ICD-10). There was increase in the yearly number of such cases and in the year 2012 it was $118 \%$ of the value in the year 2006; however the highest number of hospitalizations was recorded in the year 2009 - as it is shown in Figure 1. 


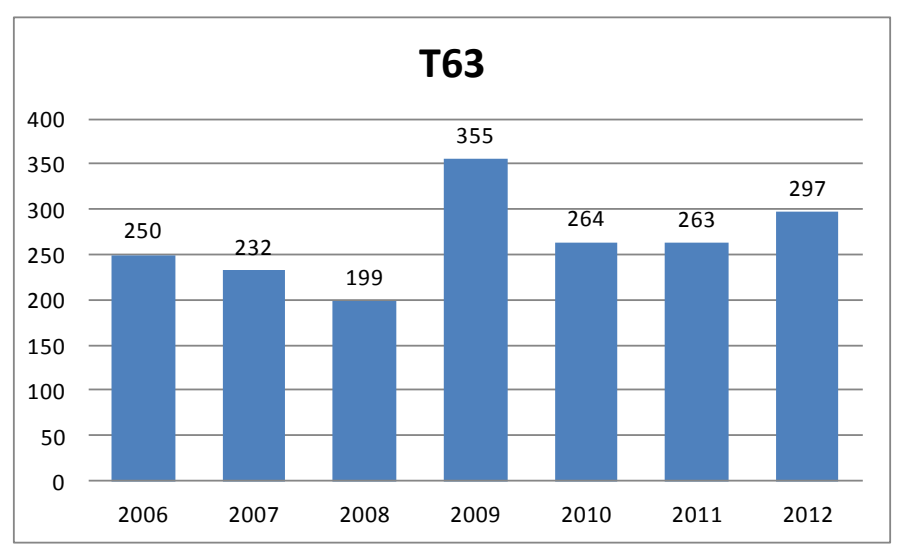

Figure1. Yearly numbers of admissions to hospitals in the Lower Silesia region of Poland in the years 20062012 due to "toxic effects of contact with venomous animals" (T63 according to ICD-10) not specified further; source: data made available by courtesy of the NFZ.

A much more striking rising trend was observed in the hospitalizations due to toxic effects of snake venom (T63.0 according to ICD-10); the yearly number of hospital admissions in the end of the analyzed period was $168,8 \%$ of its initial value - as it is illustrated in Figure 2. The total number of such cases in the whole analyzed period was 233 .

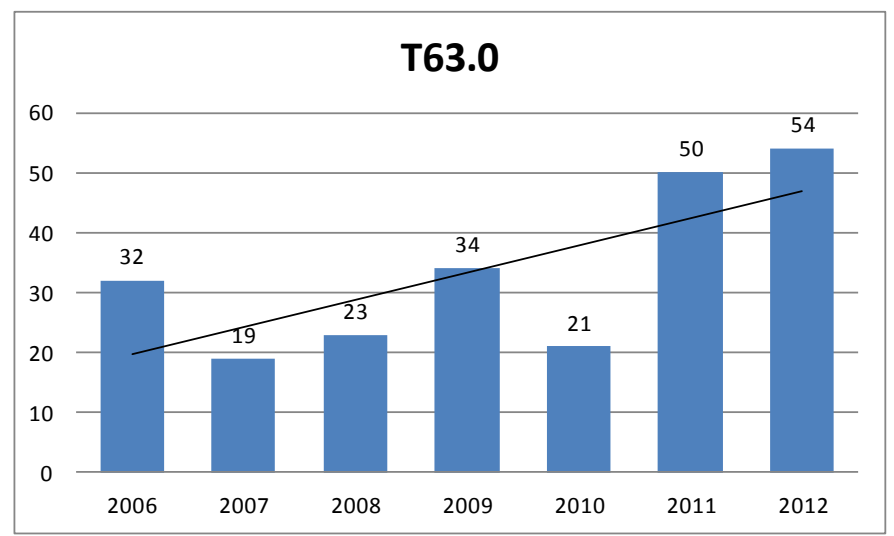

Figure2. Yearly numbers of admissions to hospitals in the Lower Silesia region of Poland in the years 20062012 due to toxic effects of snake venom (T63.0 according to ICD-10); source: data made available by courtesy of the NFZ.

The total number of hospital admissions required in the years 2006-2012 because of toxic effects caused by venoms of reptiles other than snakes (T63.1 according to ICD-10) was low with 53 cases. The yearly numbers of this kind of hospitalizations in the Lower Silesia region were alternating, however the number of hospitalizations recorded in the year 2012 was $400 \%$ of that from the year 2006 - as it is visualized in Figure 3.

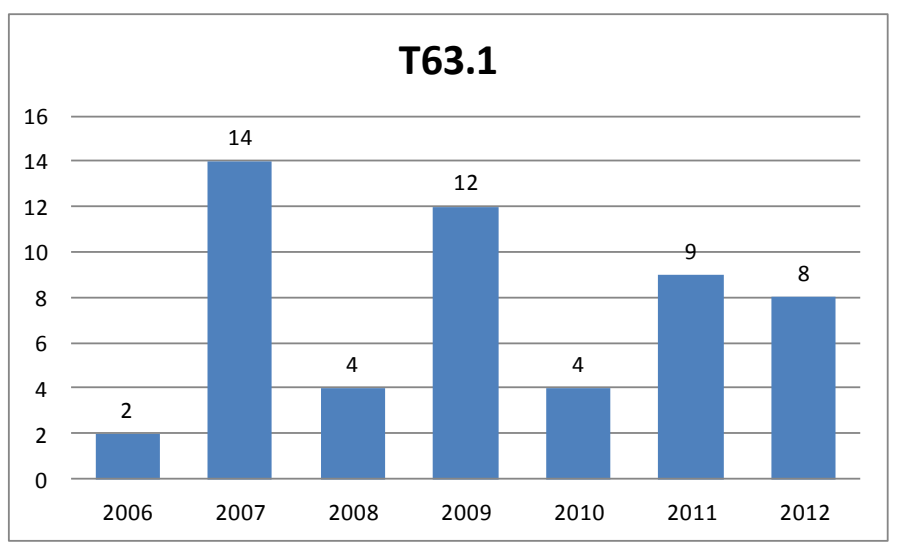

Figure3. Yearly numbers of admissions to hospitals in the Lower Silesia region of Poland in the years 20062012 due to toxic effects caused by venoms of reptiles other than snakes (T63.1 according to ICD-10); source: data made available by courtesy of the NFZ. 
Admissions to hospitals due to toxic effects caused by venom of scorpion (T63.2 according to ICD10) were casuistic in the Lower Silesia region in all analyzed years but the year 2012 when there was a big spike in the number of recorded cases - as it is shown in Figure 4; the authors did not find any reliable explanation of that rapid increase. The total recorded number of hospitalizations that belonged to this category in the analyzed time period was 21 .

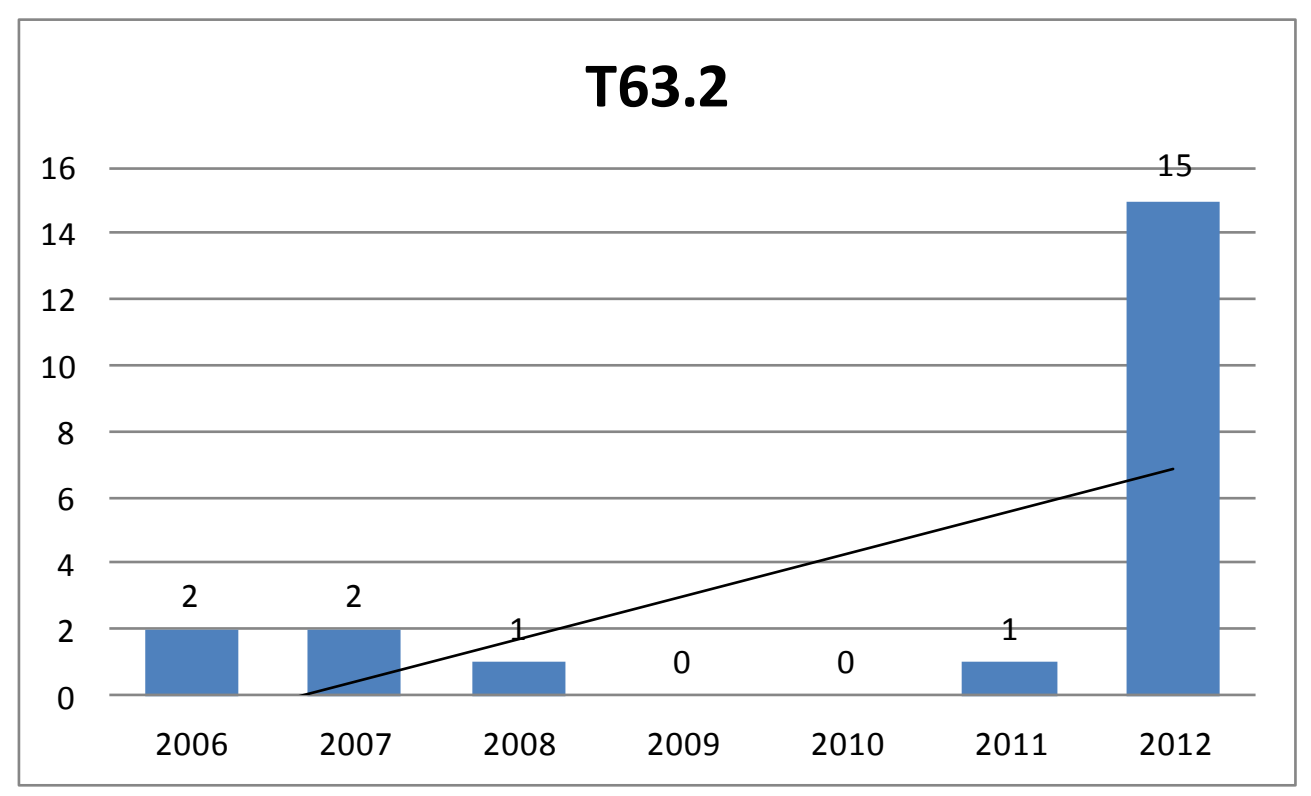

Figure4. Yearly numbers of admissions to hospitals in the Lower Silesia region of Poland in the years 20062012 due to toxic effects caused by venom of scorpion (T63.2 according to ICD-10); source: data made available by courtesy of the NFZ.

The yearly numbers of hospitalizations due to toxic effects caused by venom of spider (T63.3 according to ICD-10) varied from year to year without any recognizable trend in the changes as it is illustrated in Figure 5. The total amount of hospital admissions of this type in the analyzed period was 41 .

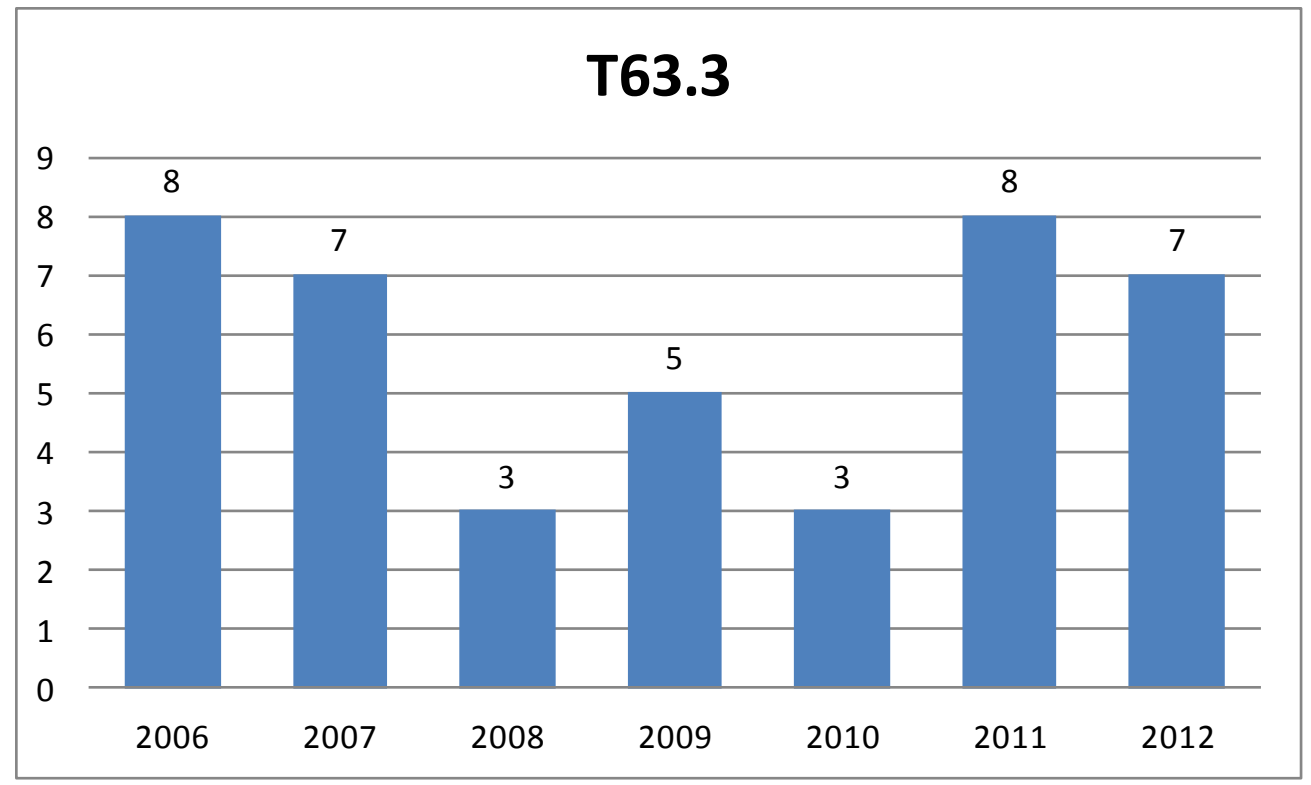

Figure5. Yearly numbers of admissions to hospitals in the Lower Silesia region of Poland in the years 20062012 due to toxic effects caused by venom of spider (T63.3 according to ICD-10); source: data made available by courtesy of the NFZ.

There were total 1405 hospitalizations associated with the toxic effects caused by venom of arthropods other than scorpions and spiders (T63.4 according to ICD-10) reported in the analyzed period. Their yearly number alternated irregularly and in the year 2012 it was 82,7\% of the year 2006 value - as it is shown in Figure 6. 


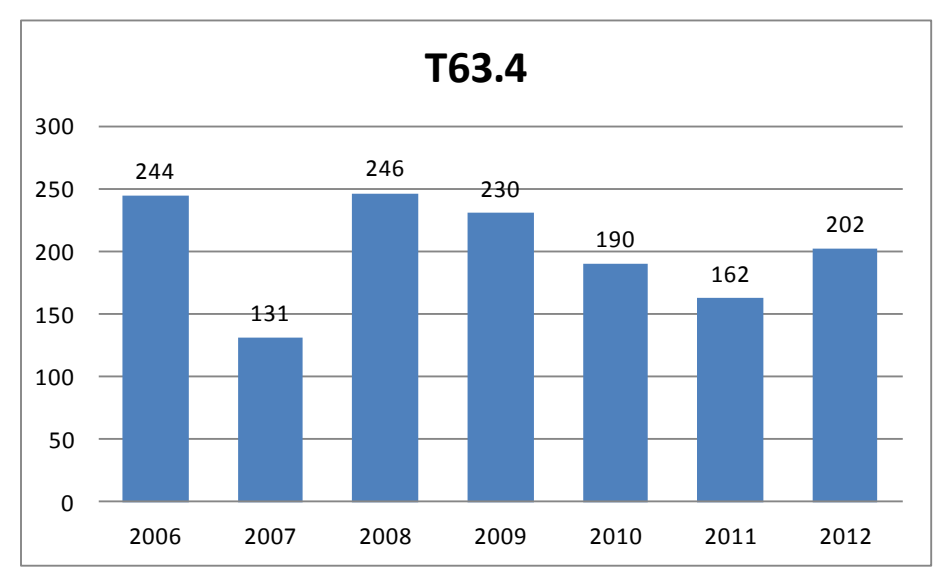

Figure6. Yearly numbers of admissions to hospitals in the Lower Silesia region of Poland in the years 20062012 due to toxic effects caused by venom of arthropods other than scorpions and spiders (T63.4 according to ICD-10); source: data made available by courtesy of the NFZ.

The number of hospital admissions due to toxic effects caused by contact with fish (T63.5 according to ICD-10) in the whole analyzed period was 69. Such cases were casuistic in the years 2006-2010 but the last 2 analyzed years there was a rapid unexplained increase in the number of recorded cases as it is visualized in Figure 7.

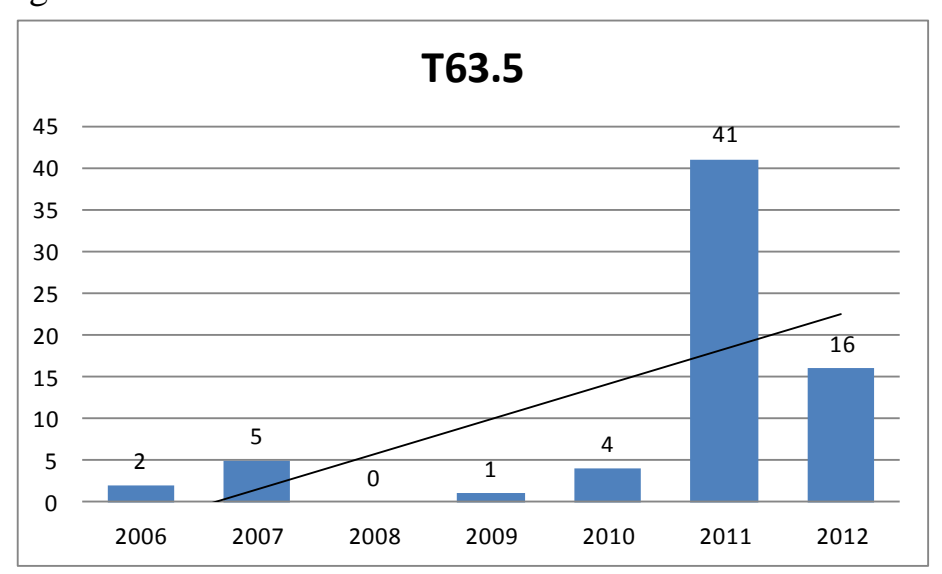

Figure7. Yearly numbers of admissions to hospitals in the Lower Silesia region of Poland in the years 20062012 due to toxic effects caused by contact with fish (T63.5 according to ICD-10); source: data made available by courtesy of the NFZ.

Cases of intoxication-related hospitalizations in the Lower Silesia region of Poland resulting from contact with marine animals other than fish (T63.6 according to ICD-10) were very rare in the analyzed period, with the recorded total number of 8 - as it is illustrated in Figure 8.

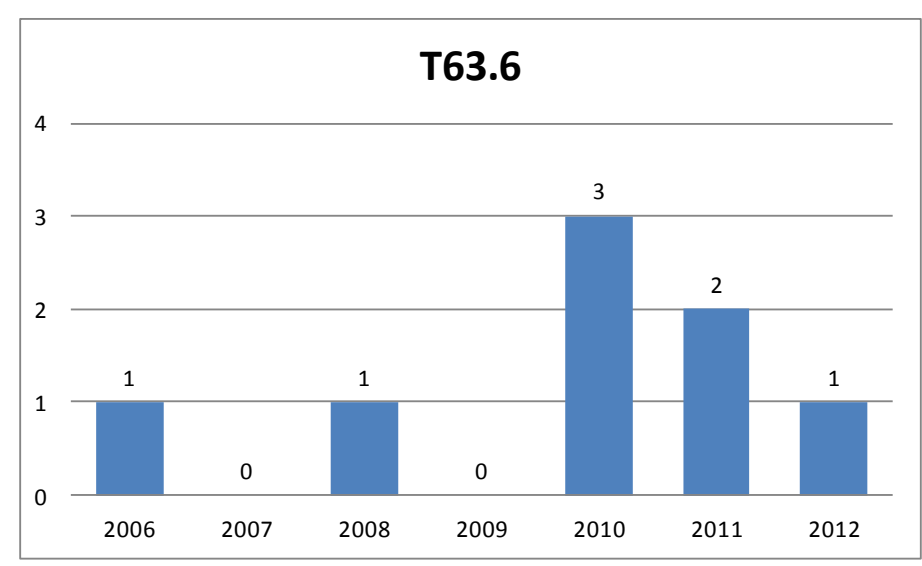

Figure8. Yearly numbers of admissions to hospitals in the Lower Silesia region of Poland in the years 20062012 due to toxic effects caused by contact with marine animals other than fish (T63.6 according to ICD-10); source: data made available by courtesy of the NFZ. 
The yearly number of admissions to the Lower Silesia region of Poland hospitals reported as resulting from toxic effects caused by contact with venomous animals other than arthropods and marine animals (T63.8 according to ICD-10) dropped rapidly in the beginning of the analyzed period to $24,2 \%$ of its initial value and later demonstrated only minor alternating - as it is shown in Figure 9. In the years 2006-2012 the total number of registered hospitalizations fitting into this category was 283. The rapid change can be attributed at least partially to the increased pressure of the NFZ to receive not general but as much as possible specific diagnoses in order to adjust payments for medical procedures properly.

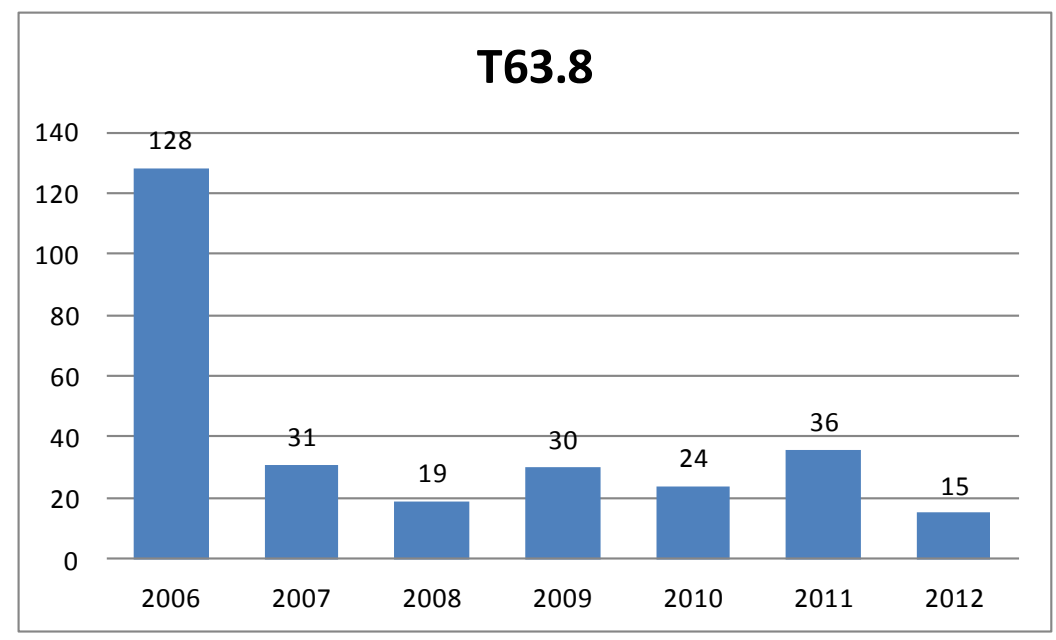

Figure9. Yearly numbers of admissions to hospitals in the Lower Silesia region of Poland in the years 20062012 due to toxic effects caused by contact with venomous animals other than reptiles, arthropods and marine animals (T63.8 according to ICD-10); source: data made available by courtesy of the NFZ.

Toxic effects caused by contact with unspecified venomous animals (T63.9 according to ICD-10) were reported as the basis of hospital admissions 564 times in the whole analyzed period. The yearly numbers were significantly alternating but a dropping trend was visible; in the 2012 the hospitalizations number was $54,7 \%$ of the value from the year 2006 - as it is illustrated in Figure 10.

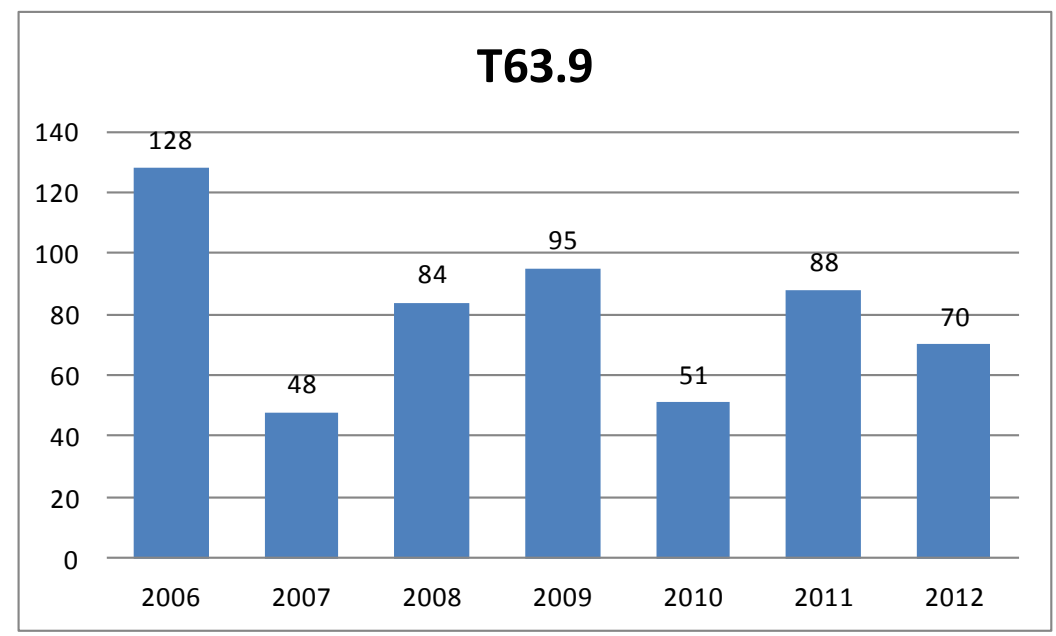

Figure10. Yearly numbers of admissions to hospitals in the Lower Silesia region of Poland in the years 20062012 due to toxic effects caused by contact with unspecified venomous animals (T63.9 according to ICD-10); source: data made available by courtesy of the NFZ.

In the Lower Silesia region of Poland contacts with hornets, wasps and bees (X23-X23.9 according to ICD-10) result in hospitalizations that are numerous in comparison to those resulting from contacts with other dangerous animals - their total number in the analyzed period was 20419. The total number of cases reported as general category only (X23 according to ICD-10) was 17259; the respective yearly numbers increased first to reach in the year $2009195,8 \%$ of the value from the year 2006 to drop rapidly in the years that followed so in the year 2012 it was only 54,2\% of the number from the beginning of the analyzed period - as it is demonstrated in Figure 11. These 
rapid changes in reported case numbers may be at least in part associated with weather conditions changing significantly from year to year that influence strongly the insects activities as the temperatures in the years 2008-2010 were significantly lower than average in Poland [20].

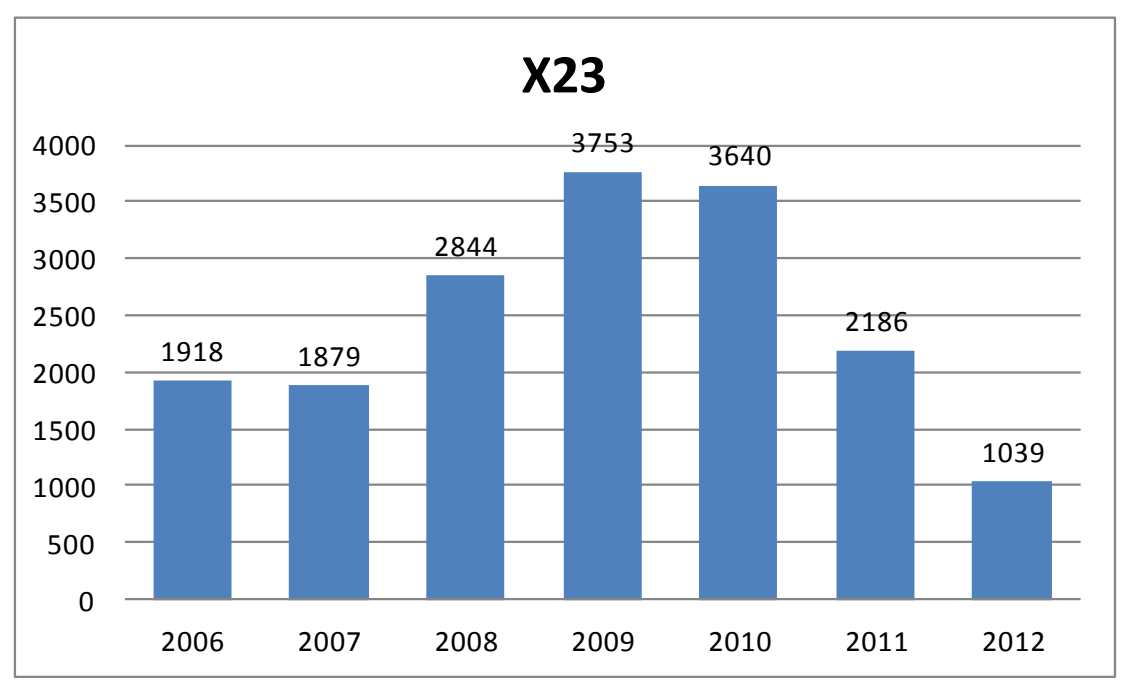

Figure11. Yearly numbers of admissions to hospitals in the Lower Silesia region of Poland in the years 2006 2012 due to "contact with hornets, wasps and bees" (X23 according to ICD-10), not otherwise specified; source: data made available by courtesy of the NFZ.

When the contacts with hornets, wasps and bees causing hospitalizations were reported as happening at home (X23.0 according to ICD-10) their total number in the analyzed years was 1517 and their yearly number demonstrated significant rising trend so that in the year 2012 it was $254,0 \%$ of the value from the beginning of the analyzed period - as it is shown in Figure 12.

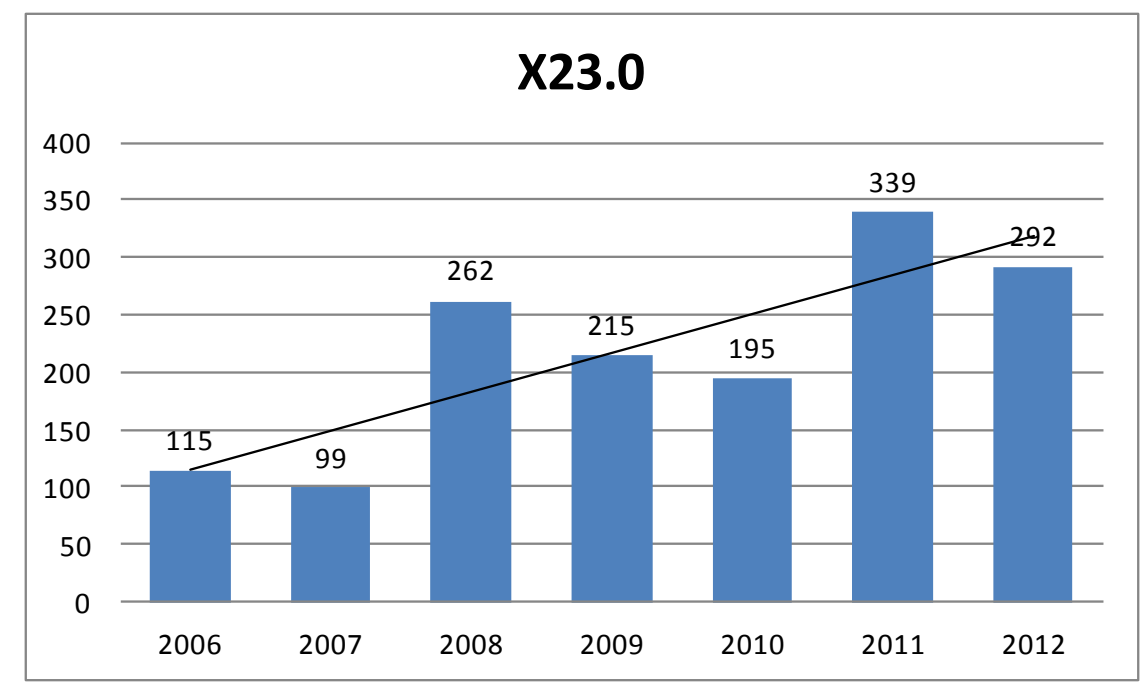

Figure12. Yearly numbers of admissions to hospitals in the Lower Silesia region of Poland in the years 20062012 due to contact at home with hornets, wasps and bees (X23.0 according to ICD-10); source: data made available by courtesy of the NFZ.

There were total 4059 cases of contacts with arthropods other than spiders, hornets, wasps, centipedes and millipedes (X25-X25.9 according to ICD-10) reported in the whole analyzed period; 208 in the year 2006 and 1009 in the year 2012 - which is $486,1 \%$ of the initial value. The total number of cases reported as not further specified general category (X25 according to ICD-10) was 3070; after first 3 years of moderate alternating their yearly number in the year 2009 jumped to $747,0 \%$ of the value from the year 2006 and later kept the new level with moderate alternating until the end of the analyzed period - as it is visualized in Figure 13. A very similar situation was observed in case of hospitalizations following such contacts that happened at home (X25.0 according to ICD-10); their yearly number demonstrated steep rising trend, in the year 2012 reaching 1228,6\% of the year 2006 value - as it is visualized in Figure 14. 


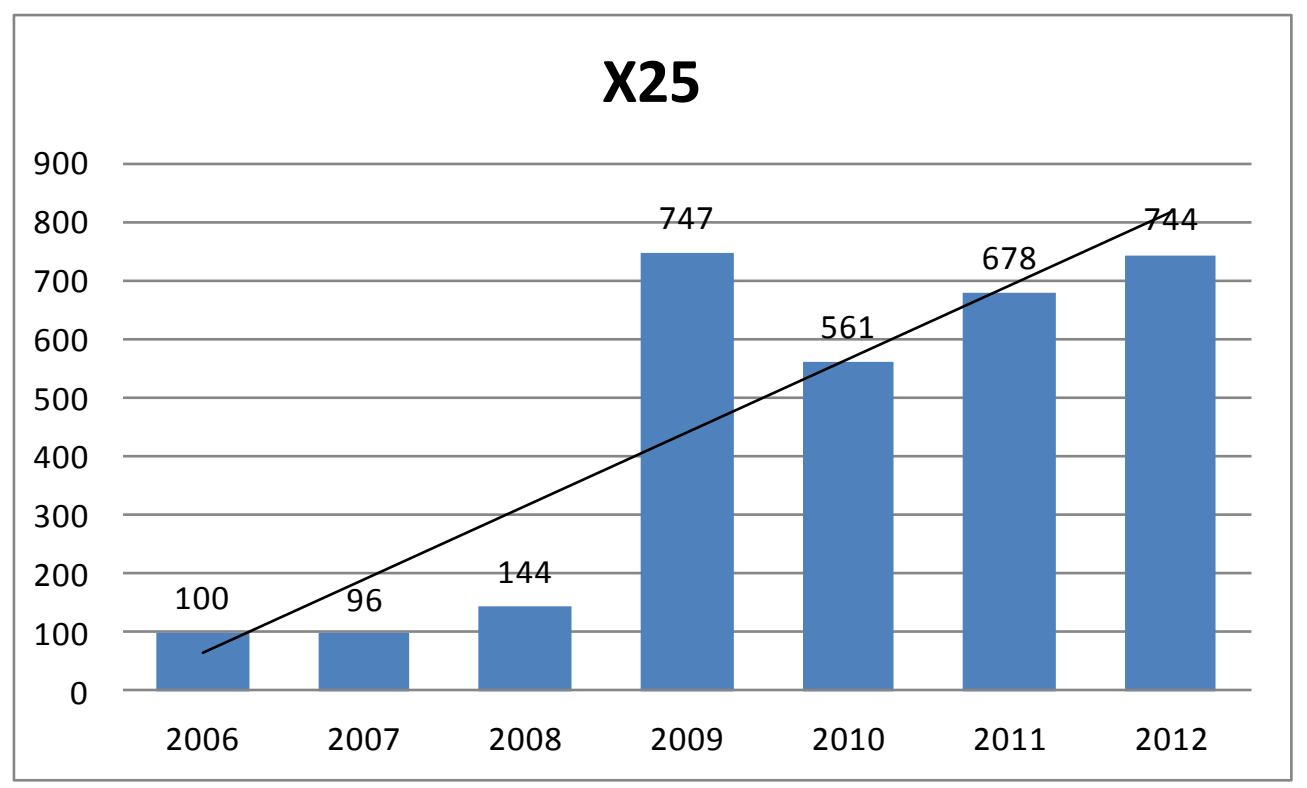

Figure13. Yearly numbers of admissions to hospitals in the Lower Silesia region of Poland in the years 20062012 due to "contact with other arthropods" than spiders, scorpions, hornets, wasps and bees, centipedes and millipedes (X25 according to ICD-10), not otherwise specified; source: data made available by courtesy of the NFZ.

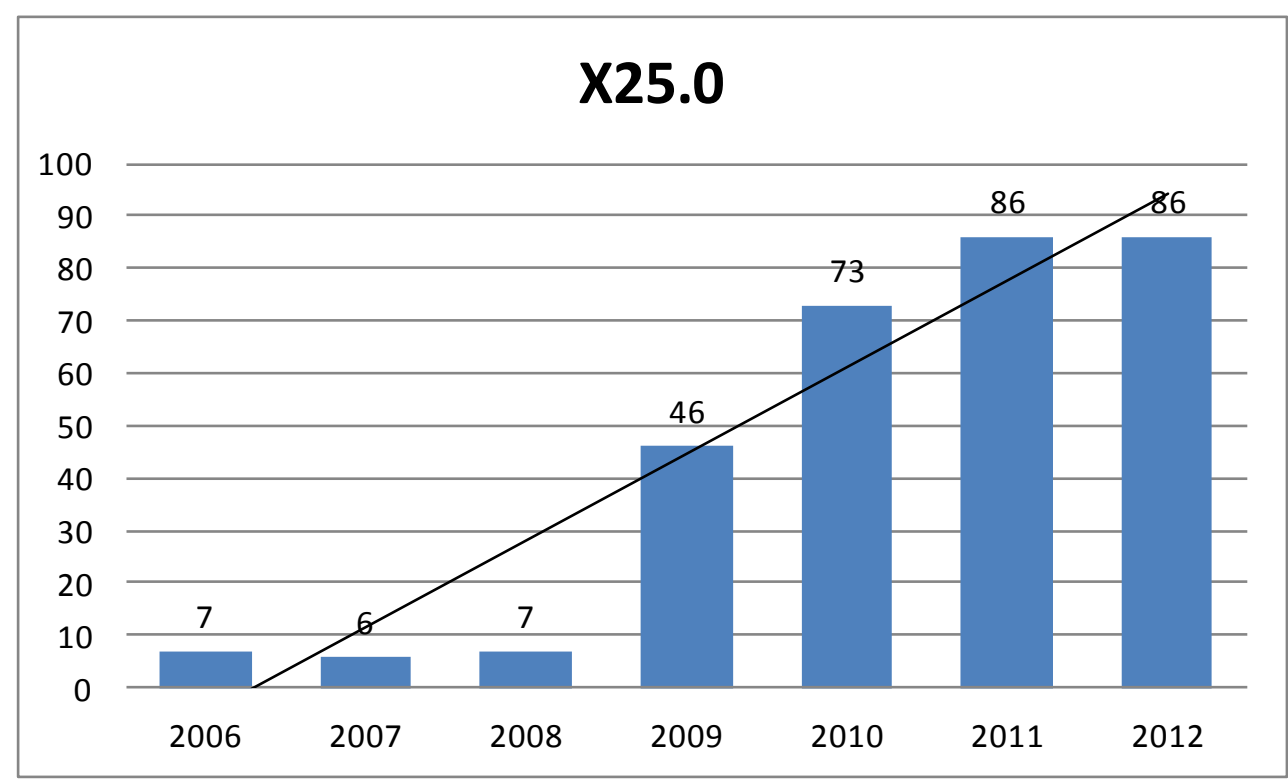

Figure14. Yearly numbers of admissions to hospitals in the Lower Silesia region of Poland in the years 20062012 due to contact at home with arthropods other than spiders, scorpions, hornets, wasps and bees, centipedes and millipedes (X25.0 according to ICD-10); source: data made available by courtesy of the NFZ.

The general structure of medical interventions due to toxic effects of contacts with animals, including envenoming (X20-X27 and T63 according to ICD-10) in the beginning and in the end of the analyzed time period is illustrated in Figure 15 and Figure 16, respectively. The most common cause of hospital admissions were contacts with arthropods, especially hornets, wasps, bees, ants and others (X23, X25 and T63.4 according to ICD-10), with the exception of contacts with venomous spiders and scorpions (X21, X22, T63.2 and T63.3 according to ICD-10), which are rare in Poland. In the year 2012 an average Pole living in the Lower Silesia region still had only minimal chance to suffer from contact with centipedes, millipedes or venomous marine animals, including fish as well as from venom of reptiles other than snakes (X24, X27, T63.5, T63.6, T63.1 according to ICD-10). It can be observed that general, unspecific classes defining contacts with venomous animals (T63, T63.8, T63.9 according to ICD-10) were still often reported by Polish physicians in the year 2012 which shall be interpreted as symptom of persisting problems with establishing the precise diagnosis and thus also an indicator of the need for improvement of medical staff diagnostic capabilities in this field. 


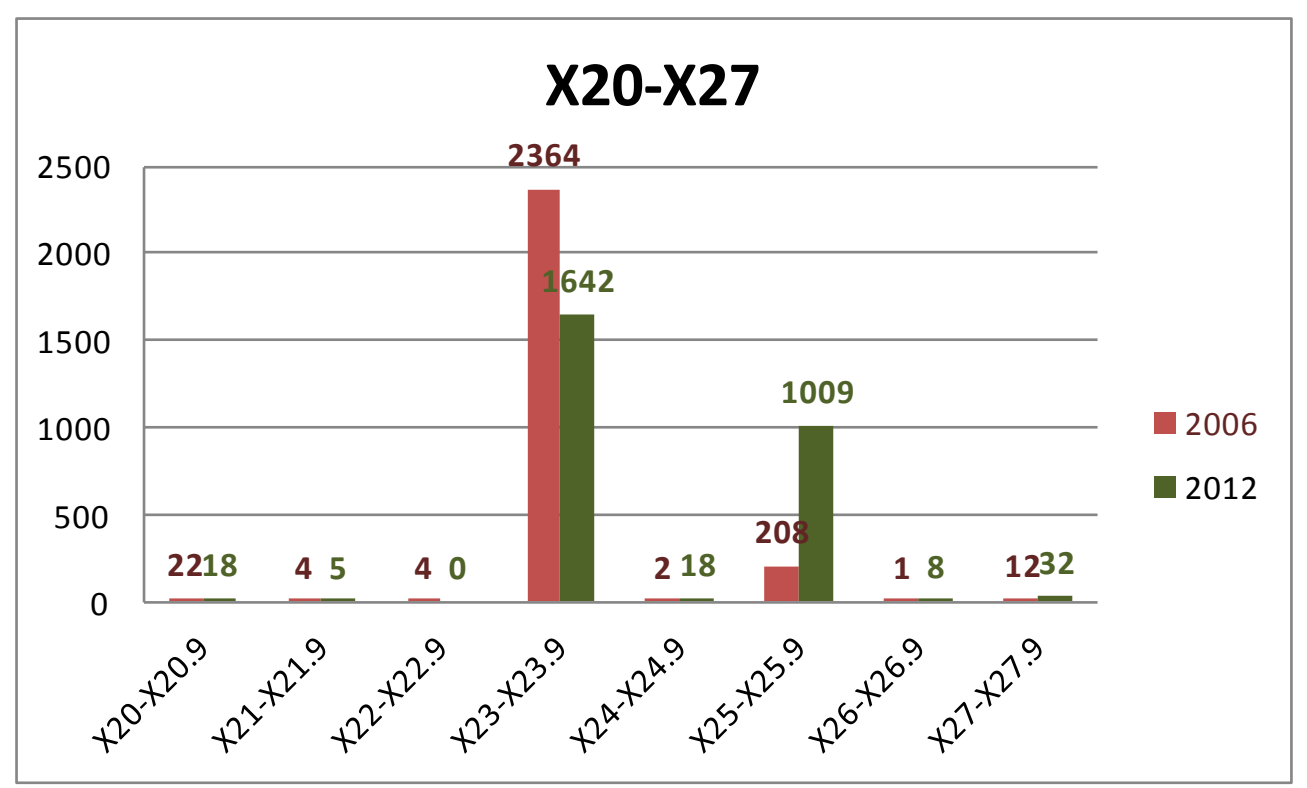

Figure15. Yearly numbers of admissions to hospitals in the Lower Silesia region of Poland in the years 20062012 due to contact with venomous animals (X20-X29 according to ICD-10); source: data made available by courtesy of the NFZ.

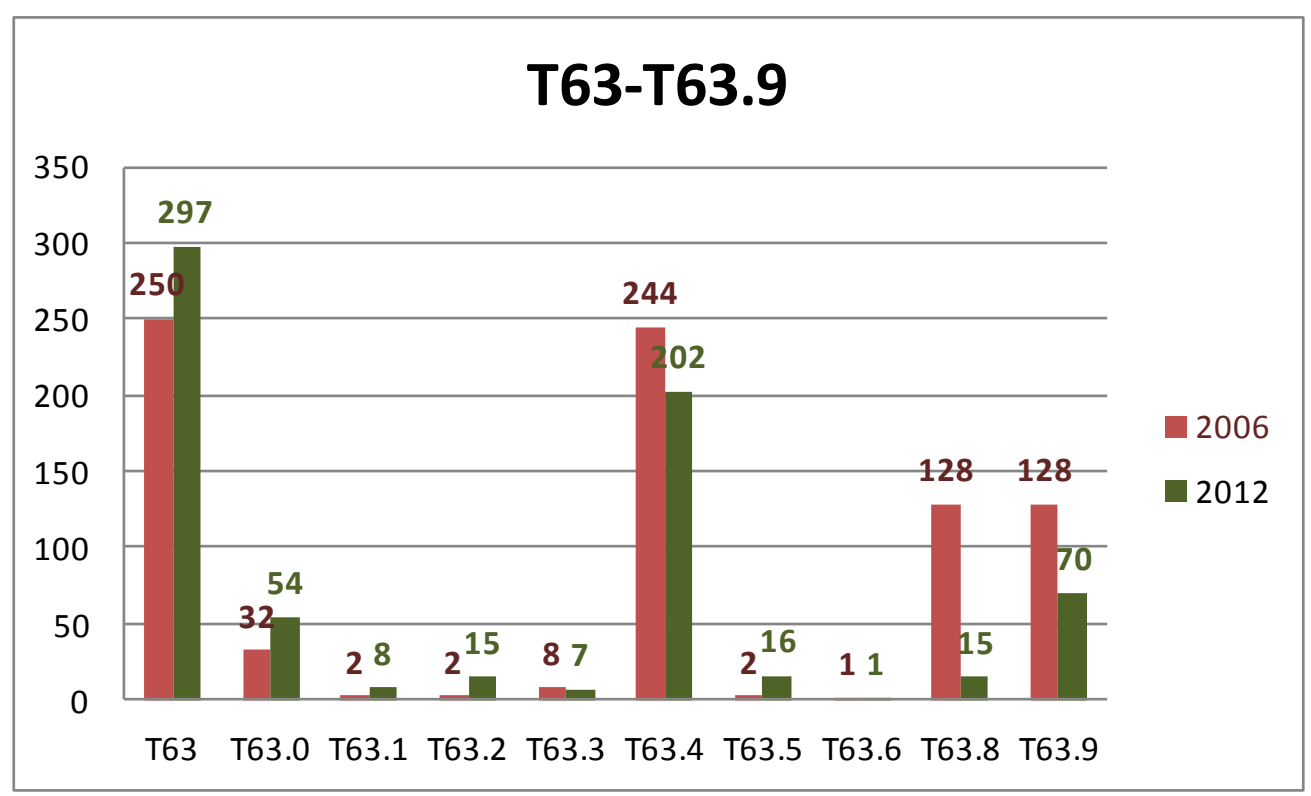

Figure16. Yearly numbers of admissions to hospitals in the Lower Silesia region of Poland in the years 20062012 due to toxic effects caused by contact with venomous animals (T63-T63.9 according to ICD-10); source: data made available by courtesy of the NFZ.

\section{Conclusion}

In the Lower Silesia region of Poland in the years 2006-2012 the most common health problems caused by contacts with animals and ending up with hospital admission were associated not with vertebrate but with arthropods - especially hornets, wasps, bees and arthropods other than scorpions and spiders. Observed differences in the occurrence rate of such cases from year to year may be associated, in a significant part at least, with changing weather conditions and resulting vegetation and animal reproduction pattern changes - however it needs further evaluation. The significant rising trend in the occurrence rate of such contacts at humans' homes may be associated with the phenomenon of re-colonization of cities by wild animals. In contrast, contacts with various venomous animals - including so widely feared by lay public contacts with snakes - were leading to hospitalizations much less often. The results of the study point at the need to increase the efforts in the field of animal monitoring and control, especially in the inhabited areas of Poland, as well as to educate people on the risks associated with contacts with even small sized animals. 
In the analyzed period there was significant rising trend in hospital admissions due to toxic effects of snake venom, which shall be attributed in the first place to the growing popularity of healthy lifestyle. Tourism and outdoor sports - especially in the forms practiced on foot - lead to mass invading by people on the more and more scarce natural habitats of the Poland's only venomous snake which results in the increased risk of snake bites. The results of the study suggest that promotion of the healthy lifestyle outdoors shall be accompanied by education on proper behavior in wild environment.

Rapid increase in hospitalizations number due to toxic effects of contact with scorpion and venomous fishes in Poland, where such species naturally do not exist at all, points at leisure activities as the major cause - especially at keeping dangerous animals as pets at private houses. The results of the study suggest the need for education of hobbyists on safety-related topics, especially at places where they can acquire dangerous exotic species.

Rapid decrease in hospital admissions due to toxic effects caused by contact with unspecified venomous animals after the year 2006 shall be interpreted as the result of introducing by the NFZ - as the major medical service payer in Poland - more strict rules of case reporting by medical institutions.

It is still very difficult to detect unknown toxic substances even using the modern sophisticated laboratory techniques - especially in an unsuspicious case of envenoming by an animal or a toxic effect resulting from a contact with an exotic animal. The results of statistical retrospective studies, like the one performed by the authors, point on the most likely intoxication causes that have to be ruled out in the first place both in clinical and forensic medicine practice. Taking into account the rising amount of these cases in Poland it is crucial for forensic medicine practitioners to receive additional education on how to gain as much information as possible from sources other than laboratory tests results, including when only available: clinical history, witnesses' reports and scene evaluation. It shall be also considered at the national level to create in Poland an animal toxic substances reference standards bank to facilitate both clinical and forensic toxicology analyses.

The results of the study draw attention to the fact that also in Poland presence of any, even minor, untypical lesions on a deceased corpse shall alert forensic medicine experts evaluating unexplained death cases and make them take into account possibility of deadly toxic effects of contacts with animals.

\section{ACKNOWLEDGMENTS}

The authors thank the NFZ for allowing them the access to the publication-relevant data.

\section{REFERENCES}

[1] Makovicky P., What Does Modern Veterinary Pathology Have to Offer; ARC Journal of Animal and Veterinary Sciences, 2015, Vol 1, Issue 1, PP 43-47

[2] Chipaux J., Epidemiology of Snakebites in Europe a Systematic Review of the Literature; Toxicon, 2012, Vol 59, Issue 1, PP 86-99; DOI: http://dx.doi.org/10.1016/j.toxicon.2011.10.008

[3] Chwaluk P., Szajewski J., Viper Bites, Przeglad Lekarski, 2000, Vol 57 Issue 10, PP: 596-599

[4] Magdalan J., Trocha M., Merwid-Lad A., Sozanski T., Zawadzki M.; Vipera berus Bites in the Region of Southwest Poland a Clinical Analysis of 26 Cases; Wilderness \& Environmental Medicine, 2010, Vol 21, Issue 2, PP 114-119 DOI: http://dx.doi.org/10.1016/j.wem.2010.01.005

[5] Boels D., Hamel J., Deguigne M., Harry P., European Viper Envenomings Assesment of Viperflav and Other Symptomatic Treatments; Clinical Toxicology, 2012, Vol 50, Issue 3, PP 189-196 DOI: http://dx.doi.org/10.3109/15563650.2012.660695

[6] Suslo R., Drobnik J., Trnka J., Kawecki J., Siuta J., Gesicki M., Fast Identification of Probable Untypical Injuries Source as an Important Factor Determining Further Medical Actions, Family Medicine \& Primary Care Review, 2014, Vol 16, Issue 4, PP 374-375

[7] Reading C., Incidence, Pathology and Treatment of Adder (Vipera berus L.) bites in man; J Accid Emerg Med 1996; 13, PP 346-351 DOI http://dx.doi.org/10.1136/emj.13.5.346

[8] Zajkowska J., Garkowski A., Pancewicz S., Vipera berus bite-epidemiology, clinical symptoms and review of treatment methods, Przeglad Epidemiologiczny, 2010, Vol 64, Issue 3, PP 387-393 
[9] Dobek R., Comparison of the Prevalence of Clinical Symptoms of Hymenoptera Venom Allergy and Its Immunological Markers in the Adult Population of Wroclaw, Poland; Posters: Insect hypersensitivity. Allergy, 2002, 57 PP 119-124 DOI http://dx.doi.org/10.1034/j.13989995.57.s73.86.x

[10] Franca F., Benvenuti L., Fan H., Santos D., Hain S., Picchi-Martins F., Cardoso L., Kimaguti A., Theakston D., Warrell D., Severe and fatal mass attacks by 'killer' bees (Africanized honey bees-Apis mellifera scutellata) in Brazil: clinicopathological studies with measurement of serum venom concentrations, QJM An International Journal of Medicine, Vol 87, Issue 5, PP 269-282

[11] Haro L., Pommier P., Envenomation: a real risk of keeping exotic house pets, Veterinary and Human Toxicology, 2003, Vol 45, Issue 4, PP 214-216

[12] Dell'Amore C. How Wild Animals Are Hacking Life in the City, http://news. Nationa lgeographic.com/2016/04/160418-animals-urban-cities-wildlife-science-coyotes/[accessed: 27.09.2016]

[13] Darsonval A., Boels D., Clerc M., Haro L., Penot-Ragon C., Moal F., Quistinic P., Lourdais O., Harry P., Creation and organization of an antivenomous serum bank in France, Presse Medicale, 2010, Vol 39, Issue 9, PP 865-870 DOI: http://dx.doi.org/10.1016/j.lpm.2010.05.018

[14] Imran M., Screening For Unknown Compounds in Forensic Toxicology, ARC Journal of Forensic Science, 2016, Vol 1, Issue 1, PP 10-12 DOI: http://dx.doi.org/10.20431/24560049.0101002

[15] Ambade V., Borkar J., Meshram S., Homicide by direct snake bite: a case of contract killing, Med Sci Law, 2012, Vol 52; PP 40-43 DOI: http://dx.doi.org/10.1258/msl.2011.011020

[16] Paulis M., Faheem A., Homicidal Snake Bite in Children, J Forensic Sci, 2010, Vol 61, No 2; PP 559-561 DOI: http://dx.doi.org/10.1111/1556-4029.12997

[17] Aggrawal A., Homicide with Snakes: a Distinct Possibility and Its Medicolegal Ramifications; Anil Aggrawal's Internet Journal of Forensic Medicine and Toxicology, 2004, Vol 4, Issue 2

[18] Drobnik J, Suslo R, Kurpas D, Trnka J.: Analysis of elderly people care system and their health needs in the Lower Silesia region; Fam.Med.Prim.Care Rev., 2010, Vol 12, Issue 2; PP 165-167

[19] Central Statistical Office of Poland Information Portal http://stat.gov.pl/statystykaregionalna/rankingi-statystyczne/ludnosc-wedlug-wojewodztw/ [accessed: 27.09.2016]

[20] Djakow P. Pogoda i Klimat, Zimne i Cieple Lata w Okresie 1979-2014, http://meteomodel. $\mathrm{pl} / \mathrm{BLOG} / \mathrm{p}=9159$ [accessed: 27.09.2016] 